\title{
Communication
}

\section{Computing Power and Sample Size for Informational Odds Ratio $^{\dagger}$}

\section{Jimmy T. Efird}

Center for Health Disparities Research and Department of Public Health, Brody School of Medicine, Greenville, NC 27858, USA; E-Mail: jimmy.efird@stanfordalumni.org; Tel.: +1-650-248-8282

$\dagger$ This paper is an extended version of paper presented at the Ninth International Symposium on Recent Advances in Environmental Health Research, Jackson, MS, USA; 16-19 September 2012.

Received: 16 November 2012; in revised form: 28 December 2012 / Accepted: 14 January 2013 / Published: 21 October 2013

\begin{abstract}
The informational odds ratio (IOR) measures the post-exposure odds divided by the pre-exposure odds (i.e., information gained after knowing exposure status). A desirable property of an adjusted ratio estimate is collapsibility, wherein the combined crude ratio will not change after adjusting for a variable that is not a confounder. Adjusted traditional odds ratios (TORs) are not collapsible. In contrast, Mantel-Haenszel adjusted IORs, analogous to relative risks (RRs) generally are collapsible. IORs are a useful measure of disease association in case-referent studies, especially when the disease is common in the exposed and/or unexposed groups. This paper outlines how to compute power and sample size in the simple case of unadjusted IORs.
\end{abstract}

Keywords: informational odds ratios; power; sample size

\section{Introduction}

A useful measure of association is the informational odds ratio (IOR) [1]. In the unadjusted case, the IOR is computed as $(\mathrm{a} / \mathrm{b}) /(\mathrm{g} / \mathrm{h})$, where $\mathrm{a}=$ number of exposed diseased individuals, $\mathrm{b}=$ number of exposed non-diseased individuals, $\mathrm{g}=$ number of diseased individuals, and $\mathrm{h}=$ number of non-diseased individuals (Table 1, Equation (1)). The IOR is equivalent to the post-exposure odds divided by the pre-exposure odds and is interpreted as an outcome measure of information gained after knowing exposure status (Equation (2)). The measure resembles the traditional odds ratio (TOR) (i.e., TOR = 
$(\mathrm{a} / \mathrm{b}) /(\mathrm{c} / \mathrm{d})$, where $\mathrm{c}=$ number of non-exposed disease individuals, $\mathrm{d}=$ number of non-exposed non-disease individuals), except that the probability terms in the denominator are not conditioned on the absence of exposure.

A key advantage of IORs is that the Mantel-Haenszel adjusted ratio estimates are collapsible (i.e., the combined crude ratio will not change after adjusting for a variable that is not a confounder) [1]. In contrast, adjusted TORs are not collapsible. IORs also are a useful and meaningful measure of association in case-reference studies of common diseases (e.g., obesity, diabetes) because their practical interpretation does not depend on estimating relative risk (RR) (i.e., rare disease assumption not required).

Prior to conducting a study it is important to determine how large a sample is needed to be reasonable confident that estimates are precise and suitable for answering a priori hypotheses. Alternatively, one may specify the sample size and then compute the study power required to reject the null hypothesis given that it is false. This paper presents simple formulas for computing power and sample size for IOR.

Table 1. A $2 \times 2$ contingency table.

\begin{tabular}{cccc}
\hline $\begin{array}{c}\text { Disease } \rightarrow \\
\downarrow \text { Exposure }\end{array}$ & D & $\overline{\mathbf{D}}$ & Total \\
\hline $\mathbf{E}$ & $\mathrm{a}=2,352$ & $\mathrm{~b}=1,600$ & $\mathrm{e}=3,952$ \\
$\overline{\mathbf{E}}$ & $\mathrm{c}=912$ & $\mathrm{~d}=1,600$ & $\mathrm{f}=2,512$ \\
Total & $\mathrm{g}=3,264$ & $\mathrm{~h}=3,200$ & $\mathrm{i}=6,464$ \\
\hline
\end{tabular}

The IOR is computed from the above $2 \times 2$ contingency table as:

$$
\mathrm{IOR}=\left(\frac{\mathrm{a} / \mathrm{b}}{\mathrm{g} / \mathrm{h}}\right)=1.44(95 \% \mathrm{CI}=1.38-1.50)
$$

where the $95 \% \mathrm{CI}$ is based on the robust variance estimate for $\log$ (IOR) [1]. The equivalence between IOR and the post-exposure odds divided by the pre-exposure odds is shown below:

$$
\mathrm{IOR}=\left(\frac{\mathrm{a} / \mathrm{b}}{\mathrm{g} / \mathrm{h}}\right)=\left(\frac{\mathrm{a} / \mathrm{g}}{\mathrm{b} / \mathrm{h}}\right)=\frac{\mathrm{P}(\mathrm{E} \mid \mathrm{D})}{\mathrm{P}(\mathrm{E} \mid \overline{\mathrm{D}})}=\left(\frac{\frac{\mathrm{P}(\mathrm{D} \mid \mathrm{E}) \mathrm{P}(\mathrm{E})}{\mathrm{P}(\mathrm{D})}}{\frac{\mathrm{P}(\overline{\mathrm{D}} \mid \mathrm{E}) \mathrm{P}(\mathrm{E})}{\mathrm{P}(\overline{\mathrm{D}})}}\right)=\left(\frac{\frac{\mathrm{P}(\mathrm{D} \mid \mathrm{E})}{\mathrm{P}(\overline{\mathrm{D}} \mid \mathrm{E})}}{\frac{\mathrm{P}(\mathrm{D})}{\mathrm{P}(\overline{\mathrm{D}})}}\right)=\frac{\text { Post-Exposure Odds }}{\text { Pre Exposure Odds }}
$$

\section{Methods}

There exist two types of error in classical statistical hypothesis testing [2]. In the current context, a rejection error (also known as a "Type I" or " $\alpha$ " error) occurs when the null hypothesis $\left(H_{0}: \mathrm{IOR}=1\right)$ versus the alternative hypothesis $\left(H_{A}: \mathrm{IOR} \neq 1\right)$ is falsely rejected, i.e., $\alpha=\mathrm{P}$ (reject $H_{0} \mid H_{0}$ is true). An acceptance error (also known as a "Type II" or " $\beta$ " error) occurs when the null hypothesis is falsely accepted, i.e., $\beta=\mathrm{P}$ (do not reject $H_{0} \mid H_{0}$ is false). The "power" of the test of hypothesis is defined as $(1-\beta)$ and denotes the probability of correctly rejecting the null hypothesis, i.e., P(reject $H_{0} \mid H_{0}$ is false). The power also conveys the likelihood that a particular research design will detect a deviation from the null hypothesis given that one exists. Another important concept in experimental design and hypothesis testing is the "sample size" of a test. Sample size denotes the number (n) of experimental units (e.g., people, animals, widgets) needed to achieve a specified power at the $\alpha$-level of statistical 
significance. Several factors influence the sample size including Type I and Type II error, and the underlying variability of the sampling distribution.

Power and sample size for IORs may be computed by a simple rearrangement of the general formulas for marginal risk ratios [3]. Letting $\mathrm{p}_{1}=$ proportion of diseased individual who are exposed, $\mathrm{p}_{0}=$ proportion of non-diseased individuals who are exposed, $\mathrm{r}=$ ratio of non-diseased to diseased individuals, $z_{\alpha / 2}=100(1-\alpha / 2)$ centile of the standard normal distribution, $Z_{\beta}=$ the standard normal deviate corresponding to $\beta=(1-$ power $)$, it follows that $Z_{\beta}=\left[n \cdot\left(p_{1}-p_{0}\right)^{2} \cdot r /(r+1) \cdot \xi \cdot(1-\xi)\right]^{1 / 2}-Z_{\alpha / 2}$ and $n=\left(Z_{\alpha / 2}+Z_{\beta}\right)^{2} \cdot \xi \cdot(1-\xi) \cdot(r+1) /\left(p_{1}-p_{0}\right)^{2} \cdot r$, where $\xi=\left(p_{1}+r \cdot p_{0}\right) /(1+r)$, and $p_{1}=p_{0} \cdot I O R$. Power then equals the probability that an observation from the standard normal distribution is less than or equal to $Z_{\beta}$. The above formulas assume a log-normal distribution for IOR and the use of a robust variance estimate for the logarithm of IOR based on the delta method [1,3]. Many commonly available statistical packages provide routines for computing power and sample size for hypothesis tests involving unadjusted RRs. These routines may be adapted to compute power and sample size for IORs by transposing the input data matrix. Results from the examples below may be used to confirm that the input matrix was properly transposed. Slight differences in the results may be due to variations in the underlying sampling distribution, algorithms and/or numerical methods used by a particular statistical package. Under general admissibility conditions, computations will converge in distribution and yield asymptotically equivalent results [4].

\section{Examples}

Assuming an equal number of diseased $\left(\mathrm{n}_{1}=100\right)$ and non-diseased $\left(\mathrm{n}_{0}=100\right)$ individuals, the plot in Figure 1 gives power for $\operatorname{IOR}=(2,3,4,5,6,7)$ for values of $\mathrm{p}_{0}$ ranging from 0.01 to 0.10 . For example, when $\mathrm{p}_{0}=0.04$, the power to detect an IOR of at least 4.0 equals $80.7 \%$ at the $\alpha=0.05$ level of statistical significance. In Figure 2, we see that 200 diseased (and 200 non-diseased) individuals are needed to be sufficiently powered $(>80 \%)$ to detect an IOR of at least 2.0 at the $\alpha=0.05$ level of statistical significance when the proportion of non-diseased individuals who are exposed equals 0.10 .

Figure 1. Power for IOR by proportion of non-diseased individuals who are exposed.

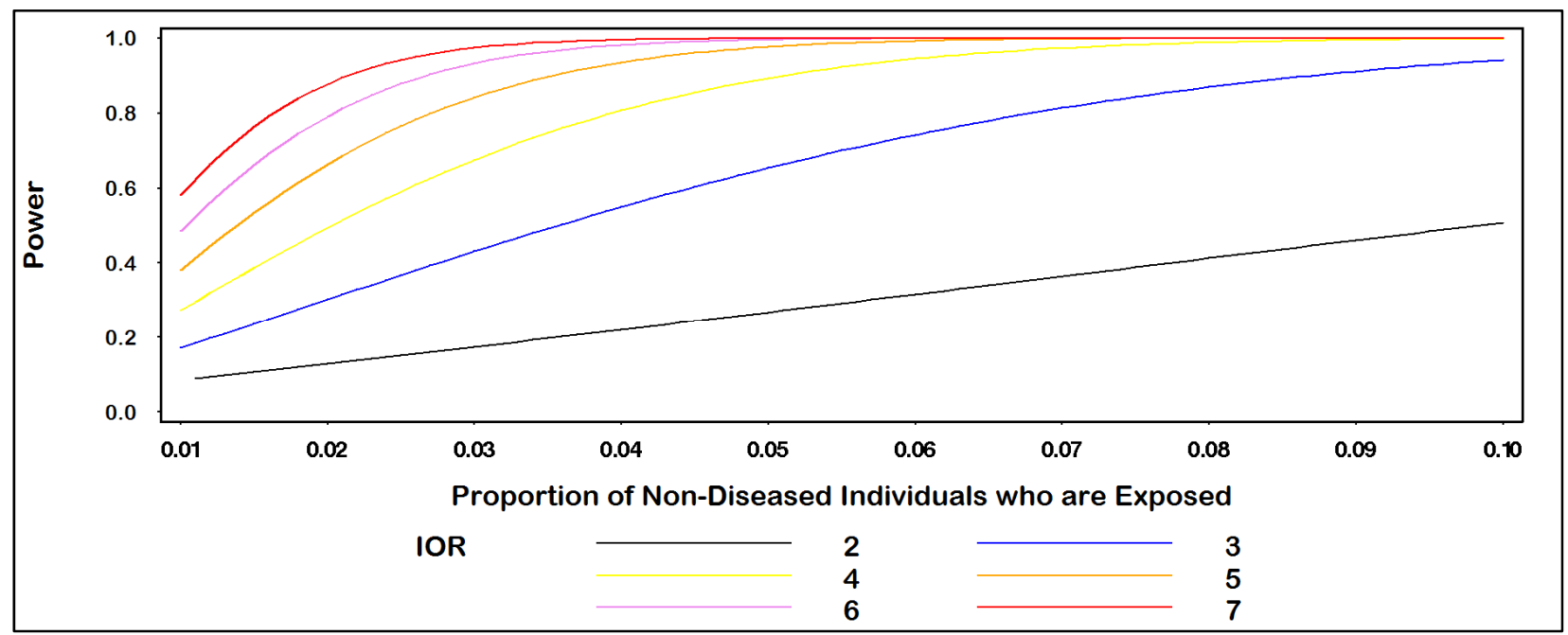

$($ Alpha $=0.05 ;$ No. non-diseased Pts. $=100 ;$ non-diseased: diseased ratio $=1.0)$. 
Figure 2. Sample size for IOR by power.

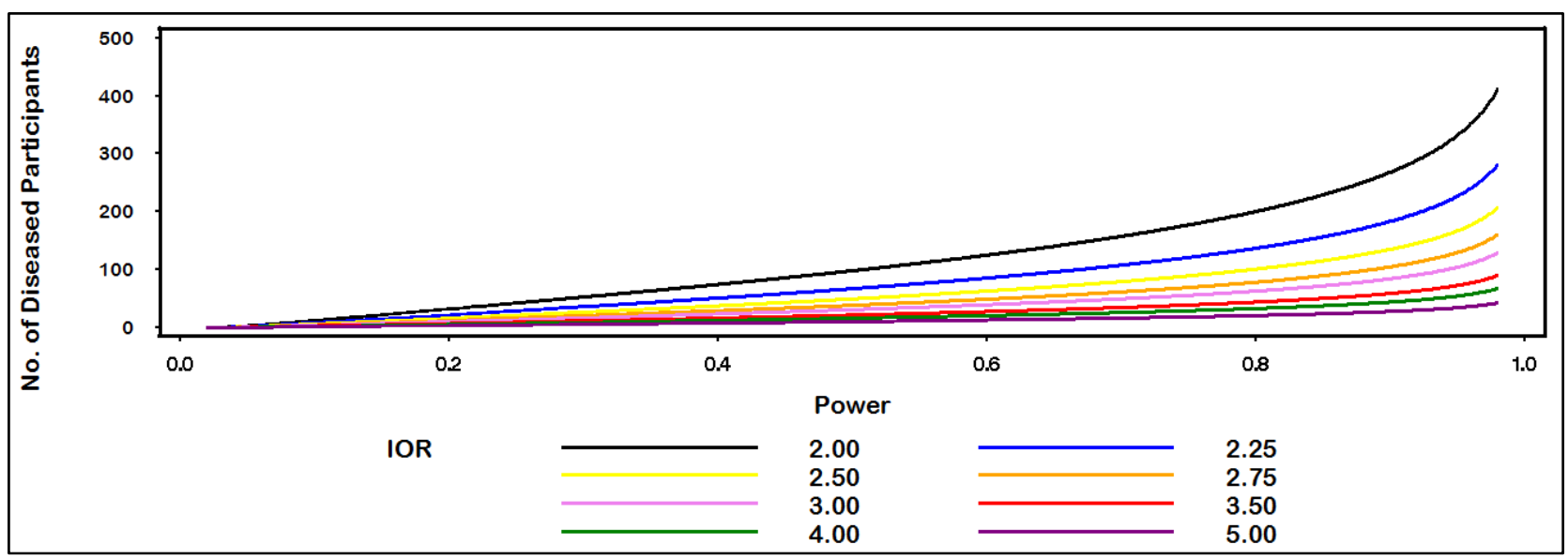

$($ Alpha $=0.05 ;$ proportion of non-diseased who are exposed $=0.10 ;$ non-diseased: diseased ratio $=1.0)$.

\section{Discussion and Conclusions}

An important property of adjusted IORs is their collapsibility and interpretability as an outcome measure of information gained after knowing exposure status (i.e., post-exposure odds divided by the pre-exposure odds). While TORs approximate RRs and collapsibility under the rare disease assumption, they lack this property in the case of retrospective studies of a common disease. The distinction of IORs versus RRs is that the former may be used in case-referent studies. This is because IORs do not depend on exposure (risk) margins but rather on column disease margins. However, because IORs still are a marginal estimate similar to RR, both estimates share the property of collapsibility.

Based on the mirror relationship of IORs and RRs as marginal measures of association, the formulas used to compute power and sample size for RRs may be simply rearranged and applied to IORs. This is a particularly useful feature in practice given the availability of software for computing the power and sample size of RR estimates.

The power and sample size formulas for IOR are based on asymptotic statistics and only should be used when the sample size is reasonably large and the sampling distribution for $\log$ (IOR) is approximately Gaussian. Similar to RRs, IORs are upwardly biased and actual power may be lower than the estimated one, at least for small sample sizes. Furthermore, the methods described for computing power and sample size apply to the simple case of unadjusted IORs and must be modified accordingly for more complex applications.

\section{Acknowledgments}

The author kindly thanks the Center for Health Disparities Research for salary support and the flexibility to explore new statistical methods.

\section{Conflicts of Interest}

The author declares no conflict of interest. 


\section{References}

1. Efird, J.T.; Lea, S.; Toland, A.; Phillips, C.J. Informational odds ratios: A useful measure of epidemiologic association. Environ. Health Insight. 2012, 6, 17-25.

2. Pagano, M.; Gauvreau, K. Principles of Biostatistics; Duxbury Press: Belmont, CA, USA, 1993; pp. 211-233.

3. Kelsey, J.L.; Whittemore, A.S.; Evans, A.S.; Thompson, W.D. Methods in Observational Epidemiology, 2nd ed.; Oxford University Press: New York, NY, USA, 1996; pp. 327-334.

4. Le Cam, L. Asymptotic Methods in Statistical Decision Theory; Springer-Verlag: New York, NY, USA, 1986; pp. 96-112.

(C) 2013 by the authors; licensee MDPI, Basel, Switzerland. This article is an open access article distributed under the terms and conditions of the Creative Commons Attribution license (http://creativecommons.org/licenses/by/3.0/). 